\title{
An easy-to-use MATLAB program (MamLand) for the assessment of landslide susceptibility using a Mamdani fuzzy algorithm
}

\begin{abstract}
In this study, landslide susceptibility mapping using a completely expert opinion-based approach was applied for the Sinop (northern Turkey) region and its close vicinity. For this purpose, an easy-to-use program, ñMamLand,ò was developed for the construction of a Mamdani fuzzy inference system and employed in MATLAB. Using this newly developed program, it is possible to construct a landslide susceptibility map based on expert opinion. In this study, seven conditioning parameters characterising topographical, geological, and environmental conditions were included in the FIS. A landslide inventory dataset including 351 landslide locations was obtained for the study area. After completing the data production stage of the study, the data were processed using a soft computing approach, i.e., a Mamdanitype fuzzy inference system. In this system, only landslide conditioning data were assessed, and landslide inventory data were not included in the assessment approach. Thus, a file depicting the landslide susceptibility degrees for the study area was produced using the Mamdani FIS. These degrees were then exported into a GIS environment, and a landslide susceptibility map was produced and assessed in point of statistical interpretation. For this purpose, the obtained landslide susceptibility map and the landslide inventory data were compared, and an area under curve (AUC) obtained from receiver operating characteristics (ROC) assessment was carried out. From this assessment, the AUC value was found to be 0.855 , indicating that this landslide susceptibility map, which was produced in a dataindependent manner, was successful.
\end{abstract}

Keyword: Mamdani fuzzy inference system; Landslide susceptibility; Geographical Information Systems (GIS); Sinop (Turkey) 